\title{
TIM4-TIM1 interaction modulates Th2 pattern inflammation through enhancing SIRT1 expression
}

\author{
TIANYONG HU ${ }^{1}$, XIAOQIN FAN ${ }^{1}$, LI MA ${ }^{1}$, JIANGQI LIU ${ }^{1}$, YUNLI CHANG ${ }^{2}$, PINGCHANG YANG ${ }^{3}$,

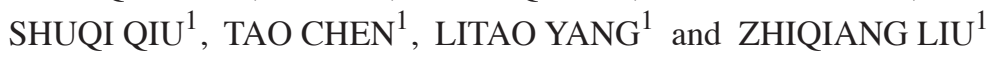

\begin{abstract}
${ }^{1}$ Immunology and Allergy Laboratory, Longgang ENT Hospital, Institute of ENT and Shenzhen Key Laboratory of ENT, Shenzhen, Guangdong 518172; ${ }^{2}$ Department of Gastroenterology, Shanghai Pudong New Area Zhoupu Hospital, Shanghai 201318;

${ }^{3}$ Shenzhen Key Laboratory of Allergy and Immunology, Shenzhen University School of Medicine and State Key Laboratory of Respiratory Disease for Allergy at Shenzhen University, Shenzhen, Guangdong 518060, P.R. China
\end{abstract}

Received December 26, 2016; Accepted September 14, 2017

DOI: $10.3892 /$ ijmm.2017.3150

\begin{abstract}
Skewed T helper 2 (Th2)-cell polarization plays a critical role in the pathogenesis of allergic inflammations; however, the underlying mechanisms require further elucidation. The aim of the present study was to investigate the mechanisms through which the interaction between T-cell immunoglobulin and mucin domain (TIM)4 and TIM1 regulates the expression of silent information regulator 1 (SIRT1) in Th 2 cells, and the role of SIRT1 in Th2-cell polarization during nasal allergic inflammation. The results demonstrated that TIM4 expression by splenic dendritic cells was increased in mice with allergic rhinitis, and the TIM4/TIM1 interaction promoted $\mathrm{CD} 4^{+} \mathrm{T}$ cells to express SIRT1 during allergic inflammation via enhancing phosphoinositide 3-kinase/Akt phosphorylation. SIRT1 then facilitated $\mathrm{CD}^{+} \mathrm{T}$-cell proliferation through downregulating the expression of Fas ligand, caspase-3 and p53 in mice with nasal allergic inflammation. In conclusion, the interaction of TIM4/TIM1 was found to promote Th2-cell proliferation through enhancing SIRT1 expression in mice with nasal allergic rhinitis.
\end{abstract}

Correspondence to: Dr Zhiqiang Liu or Dr Litao Yang, Longgang ENT Hospital, Institute of ENT and Shenzhen Key Laboratory of ENT, 3004 Longgang Avenue, Longgang, Shenzhen, Guangdong 518172, P.R. China

E-mail: liuzhiqiang05312438@126.com

E-mail: yanglitao76@163.com

Abbreviations: Th2, T helper 2; TIM, T-cell immunoglobulin and mucin domain; SIRT1, silent information regulator 1; CD, cluster of differentiation; IgE, immunoglobulin E; APCs, antigen-presenting cells; TCR, T-cell receptor; ITK, IL-2-induced T-cell kinase; PI3K, phosphoinositide 3-kinase; ELISA, enzyme-linked immunosorbent assay; AR, allergic rhinitis; OVA, ovalbumin; SIgE, OVA-specific IgE; DC, dendritic cells; CFSE, carboxyfluorescein succinimidyl ester; BSA, bovine serum albumin

Key words: allergy, T-cell immunoglobulin and mucin domain 4, T-cell immunoglobulin and mucin domain 1, T helper 2, silent information regulator 1

\section{Introduction}

The prevalence of allergic diseases has increased rapidly over the past decades, with allergic disorders affecting $>20 \%$ of the general population worldwide, including food allergies (1), allergic dermatitis (2), allergic rhinitis (AR) and asthma (3). These disorders have been classified under type I hypersensitivity. Cumulative studies have demonstrated that allergic inflammation is characterized by a T helper 2 (Th2) cell-driven immune response. IgE binds to the high-affinity receptor of $\operatorname{IgE}$ on the surface of mast cells in order to sensitize them. Re-exposure to the specific antigens cross-links the IgE/FceRI complexes on the surface of mast cells to trigger degranulation and release of inflammatory mediators $(4,5)$. Skewed Th2-cell proliferation plays a critical role in the induction of allergic inflammation by secreting interleukin (IL)-4, IL-5 and IL-13 after activation (1), while the factors involved in the maintenance of Th2-cell polarization in allergic conditions require further elucidation.

The T-cell immunoglobulin and mucin domain (TIM) family is a recently identified transmembrane molecular group; it consists of 8 members (TIM1-TIM8) in mice and 3 members (TIM1, TIM3 and TIM4) in humans (6). TIMs are involved in the regulation of innate and adaptive immune responses, such as allergy, asthma, autoimmunity and transplant tolerance $(7,8)$. TIM1, TIM3 and TIM4 have distinct functions in immune responses and they are expressed by different immune cells. Previous studies demonstrated that TIM1 was a susceptibility gene for asthma and allergy, which was preferentially expressed on activated Th2 cells; by contract, TIM4 is mainly expressed on antigen-presenting cells (APCs) (8). As one of the potent co-stimulatory signals from APCs, TIM4/TIM1 interaction facilitates Th2-cell activation and plays an important role in allergic conditions $(7,9)$. The crystal structures of TIM1 and TIM4 include an immunoglobulin variable ( $\mathrm{IgV}$ ) domain (10). TIM1 is co-localized with $\mathrm{CD} 3$ on the T-cell surface, and may be functional as part of the T-cell receptor (TCR) signaling complex during T-cell activation, possibly through IL-2-induced T-cell kinase (ITK) and phosphoinositide 3-kinase (PI3K) phosphorylation. Overexpression of TIM1 in T cells enhances the transcription of the IL-4 promoter $(8,11)$. However, further experimental research is 
required to evaluate the intracellular mechanisms of the TIM1 pathway on CD4 T-cell responses in allergic conditions.

Silent information regulator 1 (SIRT1), as a class III histone deacetylase, belongs to one of seven SIRT family members. It was previously revealed that SIRT members had conserved sequences, expressed in bacteria, mammalian and humans (12). SIRT1 deacetylates histones and other non-histone proteins, and is a multifunctional molecule involved in a variety of pathways, such as cell differentiation, apoptosis, cell aging, tumor suppression and regulation of asthmatic inflammation $(12,13)$. Notably, SIRT1 was reported to be associated with regulation of asthmatic inflammation and Th2-cell responses by inhibiting gene expression via post-translational modification of histone proteins $(14,15)$. The expression of SIRT1 in allergic conditions and the role of SIRT1 in the TIM4/TIM1 interaction-mediated $\mathrm{CD}^{+}{ }^{+} \mathrm{T}$-cell response has not been clearly determined. The aim of the present study was to investigate the expression of SIRT1 in mice with AR, and determine whether SIRT1 levels are increased in the nasal mucosa of mice with AR. In addition, the effect of the TIM4/TIM1 interaction on SIRT1 expression in splenic CD4 ${ }^{+}$ $\mathrm{T}$ cell from mice with AR was investigated, as was the effect of increased SIRT1 expression on Th2-cell polarization.

\section{Materials and methods}

Reagents. Alum and ovalbumin (OVA), carboxyfluorescein succinimidyl ester (CFSE) (21888) LY-294,002 (L9908), sirtinol (S7942) and resveratrol (R5010) were purchased from Sigma-Aldrich/Merck KGaA (Shanghai, China). The OVA-specific IgE ELISA kits were obtained from Wuhan EIAab Science (Wuhan, China). TIM4 shRNA, TIM1 shRNA, antimouse p-Akt (monoclonal; 1:200; sc-293125) and anti-mouse p53 (monoclonal; 1:100, sc-393031) were purchased from Santa Cruz Biotechnology, Inc. (Guangzhou, China). Recombinant mouse TIM4 protein was purchased from R\&D Systems (Shanghai, China). Rat allophycocyanin-anti-mouse CD4 (monoclonal; 1:200; cat. no. MCD0405), rat phycoerythrin (PE)-anti-mouse CD11c (monoclonal; 1:200; cat. no. 12-011482), rat PE-Cy7-conjugated-anti-mouse IL-4 (monoclonal; 1:200; cat. no. 25-7042-82), rat PE-conjugated-anti-mouse interferon- $\gamma$ (IFN- $\gamma$; monoclonal; 1:200; cat. no. 12-7311-82), anti-mouse CD178 (Fas ligand) FITC (monoclonal; 1:200; cat. no. 11-5911-82) and Annexin V kit were obtained from eBioscience, Inc. (Shanghai, China), rat FITC-conjugated anti-mouse TIM4 (polyclonal; 1:200; EL924747) was obtained from EterLife (Tianjin China), the caspase-3 fluorometric assay kit (K105) was obtained from Biovision (Shanghai, China), and magnetic bead-conjugated antibodies (monoclonal; $100 \mu \mathrm{l}$ antibody per $10^{8}$ total cells; 130-049-201) were purchased from Miltenyi Biotec (Shanghai, China).

Mice. A total of 48 female BALB/c mice (6-8 weeks old and weighing 18-22 g) were purchased from the Guangzhou Experimental Animal Center and were housed under pathogen-free conditions. The experimental procedures were approved by the Animal Ethics Committee at the ENT Institute of Shenzhen.

Induction of nasal allergic inflammation in mice. The AR murine model was constructed as previously described, with slight modifications (16). The mice were sensitized with OVA $(40 \mu \mathrm{g} / \mathrm{kg})$ diluted in sterile normal saline and aluminum hydroxide (alum adjuvant, $40 \mathrm{mg} / \mathrm{kg}$ ), four times by intraperitoneal (i.p.) injection on days 1, 5, 14 and 21. Intranasal challenge with OVA ( $20 \mu \mathrm{l}$ of $25 \mathrm{mg} / \mathrm{ml}$ OVA) diluted with sterile normal saline was performed daily on days 22-35. The control groups were treated with normal saline i.p. and nasal challenge. At $24 \mathrm{~h}$ after the last challenge, the mice were sacrificed and samples were collected from each mouse. The serum OVA-specific IgE was measured by ELISA. The nasal mucosa was excised for immunohistochemical analysis, and spleen mononuclear cells were isolated; $\mathrm{CD}^{+}{ }^{+} \mathrm{CD} 25^{-} \mathrm{T}$ cells and dendritic cells (DCs) were further isolated from the spleen by magnetic cell sorting with commercial reagent kits [CD4 (L3T4) MicroBeads, mouse (cat.no. 130-049-201) and CD11c MicroBeads UltraPure, mouse (cat. no. 130-108-338) (both from Miltenyi Biotec)] following the manufacturer's instructions. The purity of the CD4 ${ }^{+}$CD25 T cells and DCs was $>98 \%$, as determined by flow cytometry.

Animal groups. The 48 mice were randomly divided into control and AR groups ( $\mathrm{n}=24$ each), with 4 subgroups in each category ( $n=6$ per subgroup). One subgroup from each group was used to examine: i) Splenic mononuclear cells for the frequency of $\mathrm{CD} 4^{+} \mathrm{IL}-4^{+} \mathrm{T}$ cells and $\mathrm{CD} 4^{+} \mathrm{IFN}-\gamma^{+} \mathrm{T}$ cells by fluorescence-activated cell sorting (FACS; Fig. 1); ii) splenic DCs for TIM4 expression and CD4 ${ }^{+}$T-cell proliferation (Fig. 2); iii) nasal mucosa tissues and splenic $\mathrm{CD}^{+} \mathrm{T}$ cells (Fig. 3); and iv) splenic mononuclear cells and $\mathrm{DCs} C D 4^{+} \mathrm{T}$ cells to determine the role of SIRT1 in their proliferation and measure the Fas ligand (FasL), caspase-3 and p53 expression (Figs. 4 and 5).

Histology. The nasal mucosa samples were collected and fixed in $4 \%$ paraformaldehyde overnight and processed for paraffin embedding. Sections $(4 \mu \mathrm{m})$ were prepared and stained with hematoxylin and eosin for detection of inflammatory cell infiltration.

ELISA. The serum levels of OVA-specific IgE were measured with purchased reagent kits (Wuhan EIAab Science) following the manufacturers' instructions.

Gene silencing. The TIM4 gene in splenic DCs and the TIM1 gene in $\mathrm{CD}^{+}{ }^{+} \mathrm{T}$ cells were knocked down by RNA interference with reagent kits [Lipofectamine ${ }^{\mathrm{TM}} 3000$ Transfection Reagent (L3000015); Invitrogen, Shanghai, China] following the manufacturer's instructions. The effect of gene silencing was assessed by western blotting.

Flow cytometry. Cells were collected from the culture and fixed with $1 \%$ formaldehyde and $0.1 \%$ Triton X-100 (and permeabilization buffer, if necessary) for $30 \mathrm{~min}$ at $4^{\circ} \mathrm{C}$, washed with $1 \%$ bovine serum albumin (BSA)/phosphate-buffered saline (PBS) 3 times, and blocked for $30 \mathrm{~min}$ at $4^{\circ} \mathrm{C}$ with $1 \%$ BSA. Cells were incubated with the fluorescence-labeled antibodies for $1 \mathrm{~h}$ at room temperature. After washing with PBS, the cells were analyzed with a flow cytometer (FACSCanto II; BD Biosciences, Franklin Lakes, NJ, USA).

Western blot analysis. Total protein was extracted from the cells with a protein extraction buffer $\left[\right.$ M-PER ${ }^{\mathrm{TM}}$ Mammalian 

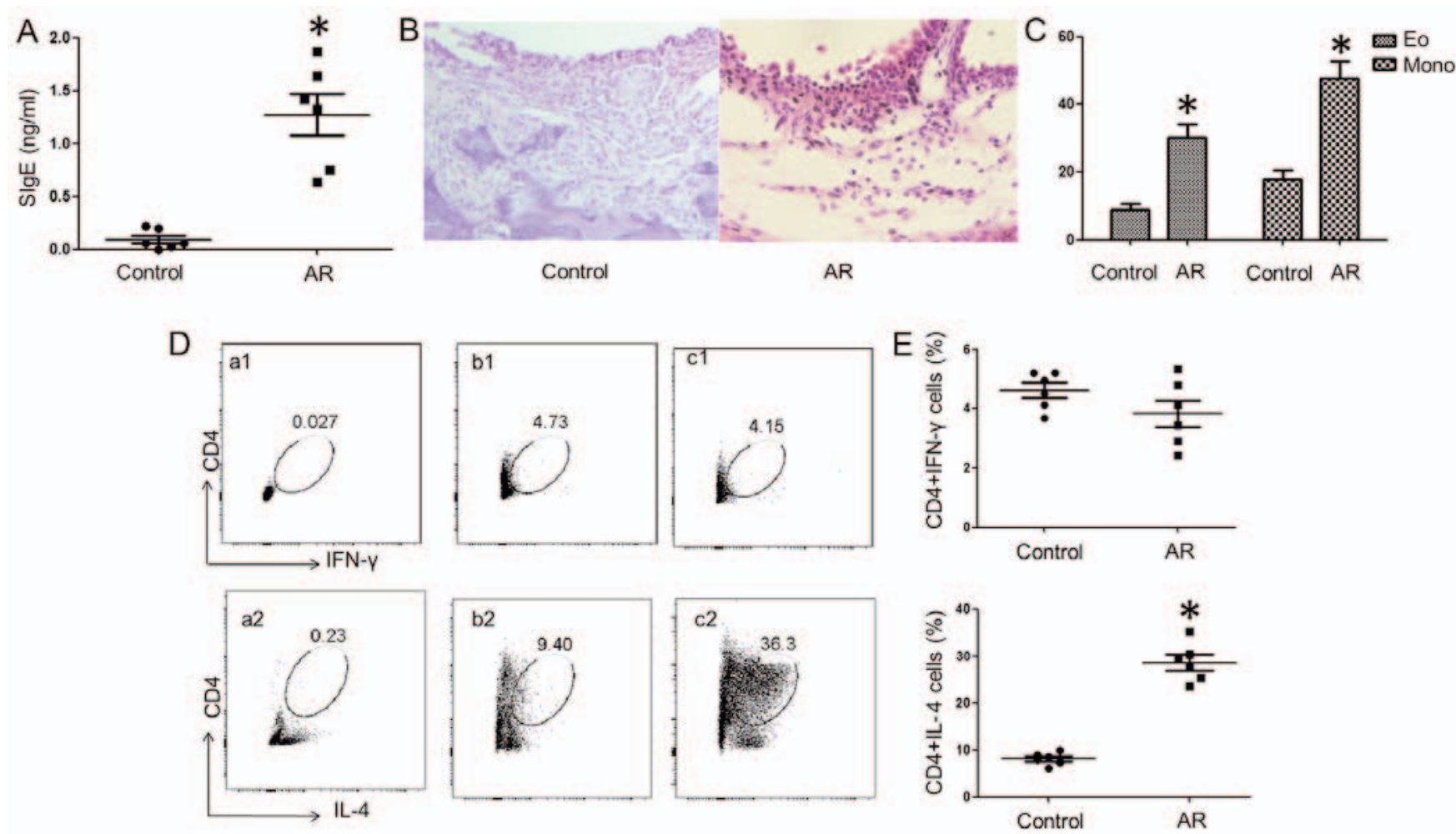

Figure 1. Induction of a mouse model with allergic inflammation. Samples were collected from mice with AR and control mice. (A) The dot plots indicate the levels of OVA-specific IgE (SIgE) in the serum. (B) Histopathological images of the nasal mucosa. Hematoxylin and eosin staining; magnification, x200. (C) The bars indicate the counts of eosinophils (Eo) and mononuclear (Mono) cells in the nasal mucosa. (D) The scatter plots indicate the frequency of CD4+ IL-4 ${ }^{+} \mathrm{T}$ cells and $\mathrm{CD} 4{ }^{+}$IFN $-\gamma^{+}$T cells in splenic Mono cells. (a1 and a2) Isotype control; (b1 and b2) control; (c1 and c2) AR. (E) The dot plots represent the summarized frequencies of (D). The data are presented as mean \pm standard deviation. ${ }^{*} \mathrm{P}<0.05$ vs. the control group. AR, allergic rhinitis; OVA, ovalbumin; IL, interleukin; IFN, interferon.

Protein Extraction Reagent (cat. no. 78503); Thermo Fisher, Shanghai, China]. The protein concentration was measured by the bicinchoninic acid method. After denaturing, the samples were loaded in duplicate onto a $10 \%$ sodium dodecyl sulfate polyacrylamide gel; proteins were separated by electrophoresis and transferred onto a nitrocellulose membrane. The membrane was blocked by $5 \%$ skimmed milk for $30 \mathrm{~min}$ and incubated with the primary antibodies $(50-100 \mathrm{ng} / \mathrm{ml})$ followed by the secondary antibodies. The immune complex on the membrane was developed by enhanced luminol-based chemiluminescence and the results were photographed using the UVP BioSpectrum Imaging system (BioSpectrum, Upland, CA, USA).

Reverse transcription-quantitative polymerase chain reaction $(R T-q P C R)$. Nasal mucosa was removed and total RNA was extracted using an RNeasy mini kit (Qiagen, Inc., Valencia, CA, USA). A total of $1 \mu \mathrm{g}$ RNA was reverse-transcribed into cDNA with the IScript ${ }^{\mathrm{TM}}$ cDNA synthesis kit (Bio-Rad Laboratories, Inc., Hercules, CA, USA) according to the manufacturer's protocol. The resulting complementary DNA was then subjected to qPCR on the MiniOpticon PCR system. The primers used in the experiments were as follows: SIRT1: Forward, ctgttgaccgat ggactcct and reverse, gccacagcgtcatatcatcc; $\beta$-actin: Forward, gtgggaatgggtcagaagga and reverse, tcatctttcacggttggcc. The amplification protocol was performed as follows: 1 cycle at $98^{\circ} \mathrm{C}$ for $1 \mathrm{~min}$ followed by 40 cycles at $98^{\circ} \mathrm{C}$ for $10 \mathrm{sec}, 55^{\circ} \mathrm{C}$ for $20 \mathrm{sec}$, and $72^{\circ} \mathrm{C}$ for $30 \mathrm{sec}$. The relative SIRT1 gene expression compared with a housekeeping gene was analyzed using the comparative quantification cycle method.
Statistical analysis. All values are presented as mean \pm standard deviation of a minimum of three independent experiments. The values were analyzed by one-way analysis of variance, followed by Tukey's test for multiple comparisons. $\mathrm{P}<0.05$ was considered to indicate statistically significant differences.

\section{Results}

Establishment of mouse model with nasal Th2 type inflammation. A mouse model of AR was developed as previously reported (16). Compared with the control group, mice sensitized with OVA and alum (AR group) had more denuded skin around the nose and a higher number of scratching events (data not shown). OVA-specific IgE (SIgE) was detected in the serum (Fig. 1A). Inflammatory cell infiltration in the nasal mucosa was observed in the AR group (Fig. 1B and C). As shown by flow cytometry, more IL- $4^{+} \mathrm{CD} 4^{+} \mathrm{T}$ cells and fewer IFN $-\gamma^{+} \mathrm{CD} 4 \mathrm{~T}$ cells were detected in the spleens of the allergy group compared with the control group (Fig. 1D).

The TIM4/TIM1 interaction promotes Th2-cell proliferation. Splenic DCs were isolated from AR mice and control mice. The DCs were pulsed with OVA $(10 \mathrm{ng} / \mathrm{ml})$ in the culture for 3 days. TIM4 expression was detected in DCs by confocal microscopy (Fig. 2A). DCs from the AR group exhibited higher expression of TIM4 compared with the control group. Splenic $\mathrm{CD} 4^{+} \mathrm{T}$ cells were isolated and co-cultured with DCs in the presence of OVA $(10 \mathrm{ng} / \mathrm{ml})$ for 3 days and analyzed by flow cytometry. $\mathrm{CD}^{+} \mathrm{T}$ cell proliferation from the AR group 
A

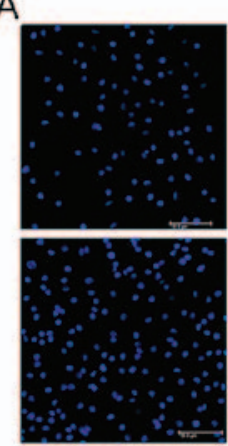

DAPI
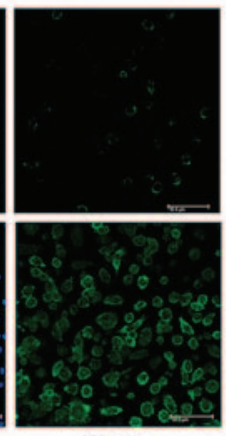

TIM4
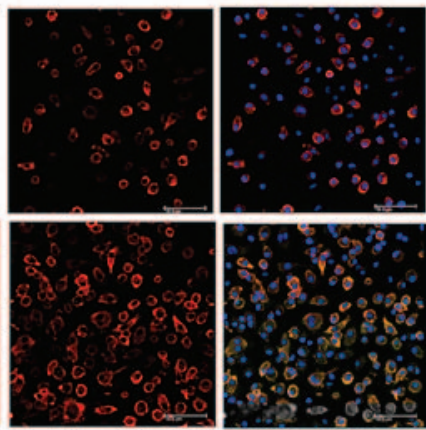

$\mathrm{CD} 11 \mathrm{c}$

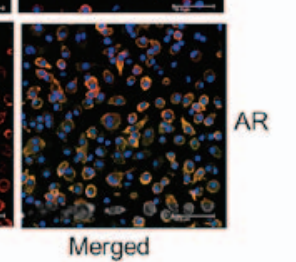

$\mathrm{B}$

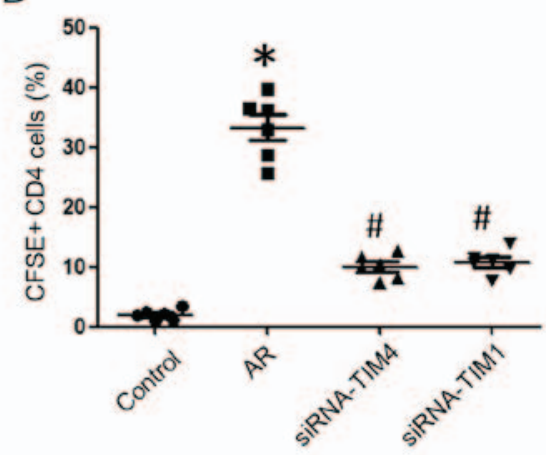

C

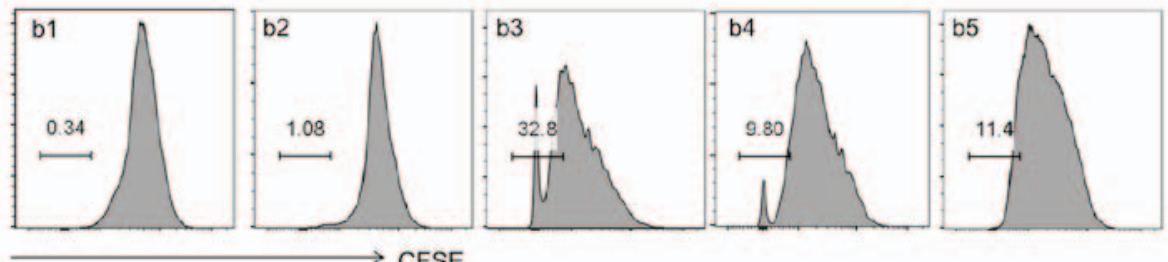

Figure 2. T-cell immunoglobulin and mucin domain (TIM)4/TIM1 interaction enhanced CD4 ${ }^{+}$T-cell proliferation. (A) Confocal images of TIM4 expression on splenic dendritic cells (DCs) following exposure to ovalbumin (OVA) in the culture for 3 days. (B) The dot plots show the summarized frequencies of (C), with data presented as mean \pm standard deviation; ${ }^{P} \mathrm{P}<0.05$ vs. the control group and ${ }^{\#} \mathrm{P}<0.05$ vs. the AR group. (C) The gated histograms indicate the frequency of proliferating $\mathrm{CD}^{+} \mathrm{T}$ cells following exposure to OVA and DCs in the culture for 3 days. (b1) Carboxyfluorescein succinimidyl ester (CFSE)-positive control, (b2) CD4 ${ }^{+} \mathrm{T}$ cells from the control group; (b3) cells from the allergic rhinitis (AR) group; (b4) cells from the AR group cultured with DCs (blocked by siRNA-TIM4); (b5) cells from the AR group blocked by siRNA-TIM1.

A

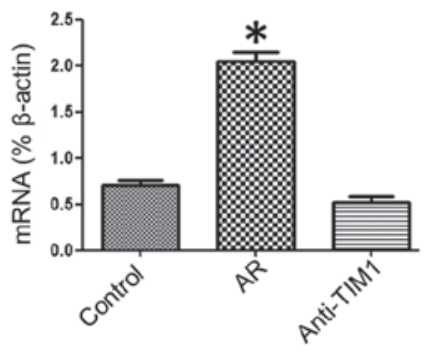

B
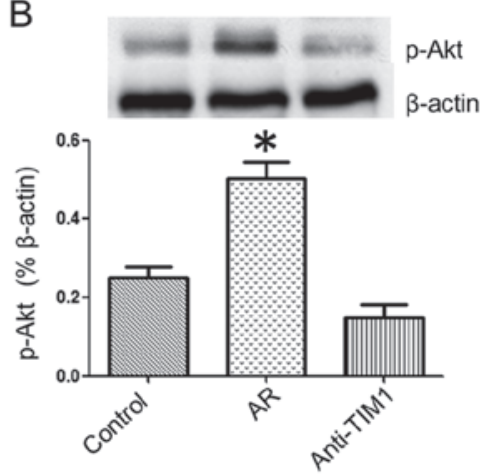

C
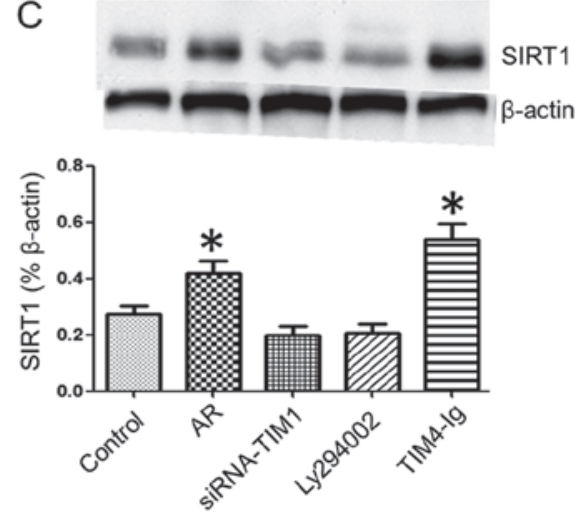

Figure 3. T-cell immunoglobulin and mucin domain (TIM)4/TIM1 interaction increases silent information regulator 1 (SIRT1) expression in splenic CD4+ T cells. The RNA expression of SIRT1 in the nasal mucosa was evaluated by quantitative polymerase chain reaction. Splenic mononuclear cells in each group were collected and cultured with ovalbumin $(10 \mathrm{ng} / \mathrm{ml})$ for 3 days. CD4 ${ }^{+} \mathrm{T}$ cells were isolated by microbeads and SIRT1 expression was assessed by western blot analysis. (A) The bars indicate the SIRT1 mRNA levels in the nasal mucosa. (B and C) The immune blots show the levels of (B) phosphorylated Akt (p-Akt) and (C) the SIRT1 protein in splenic $\mathrm{CD}^{+} \mathrm{T}$ cells. The groups were annotated below the graphs. The data are presented as mean \pm standard deviation. ${ }^{*} \mathrm{P}<0.05$ vs. the control group. Ly294002, phosphoinositide 3-kinase/Akt inhibitor; AR, allergic rhinitis.

was markedly increased, which was inhibited by gene silencing of TIM4 in DCs and TIM1 in CD4 ${ }^{+} \mathrm{T}$ cells (Fig. 2B and C). The results suggest that the TIM4/TIM1 interaction facilitates Th2-cell proliferation in AR mice.

The TIM4/TIM1 interaction modulates SIRT1 expression in splenic $C D 4^{+}$T cells. To determine the role of the TIM4/TIM1 interaction in the expression of SIRT1 in DCs, samples of splenic DCs were analyzed by RT-qPCR. It was observed that the SIRT1 mRNA expression was increased in the nasal mucosa of AR mice, which was blocked by caudal vein injection of
anti-TIM1 blocking antibody (50 $\mu \mathrm{g} /$ mouse) (Fig. 3A). Next, the effect of the TIM4/TIM1 interaction on SIRT1 expression was evaluated in $\mathrm{CD}^{+} \mathrm{T}$ cells. Splenic mononuclear cells in each group were collected and cultured with OVA $(10 \mathrm{ng} / \mathrm{ml})$ for 3 days, $\mathrm{CD}^{+} \mathrm{T}$ cells were isolated by microbeads, and SIRT1 expression in $\mathrm{CD}^{+} \mathrm{T}$ cells was assessed by western blot analysis. The results demonstrated that Akt phosphorylation was increased in splenic $\mathrm{CD} 4^{+} \mathrm{T}$ cells from the AR group and it was inhibited by TIM1 gene silencing (Fig. 3B). The SIRT1 expression was higher in splenic $\mathrm{CD}^{+} \mathrm{T}$ cells from the AR group compared with the control group, while 
A

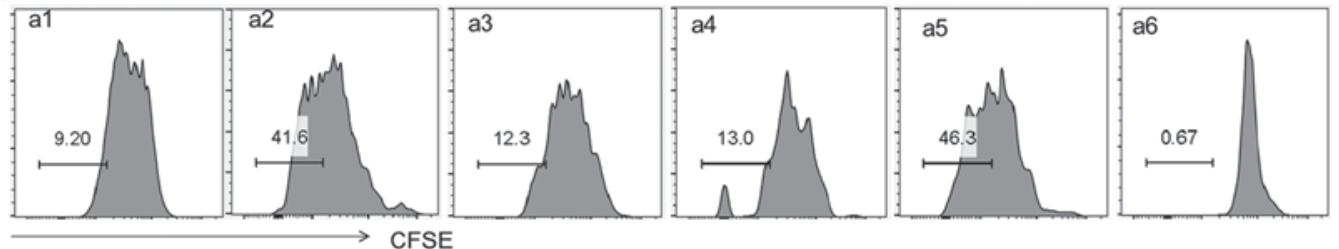

B
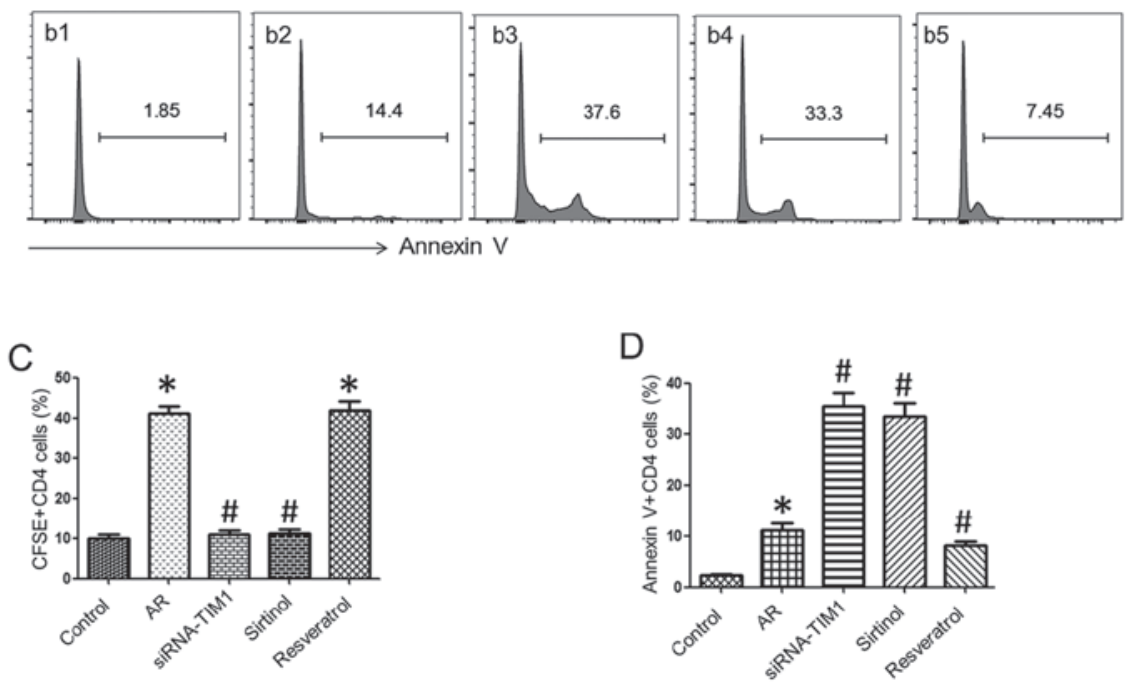

Figure 4. Silent information regulator 1 (SIRT1) regulates $\mathrm{CD} 4^{+} \mathrm{T}$-cell proliferation and apoptosis. The gated histograms indicate (A) splenic $\mathrm{CD} 4{ }^{+} \mathrm{T}-\mathrm{Cell}$ proliferation and (B) splenic CD4+ T-cell apoptosis. (a1 and b1) Cells from the control group; (a2 and b2) cells from the allergic rhinitis (AR) group; (a3 and b3) cells from the $\mathrm{AR}$ group and $\mathrm{CD} 4^{+} \mathrm{T}$ cells were blocked by siRNA-T-cell immunoglobulin and mucin domain (TIM)1; (a4 and b4) cells from the AR group and $\mathrm{CD}^{+} \mathrm{T}$ cells were cultured with sirtinol (SIRT1 antagonist); (a5 and b5) cells from the AR group and CD4 ${ }^{+} \mathrm{T}$ cells were stimulated with resveratrol (SIRT1 agonist); (a6) carboxyfluorescein succinimidyl ester (CFSE)-positive control. (C) The bars show the summarized frequencies of (A). (D) The bars show the summarized frequencies of (B). Data are presented as mean \pm standard deviation. ${ }^{*} \mathrm{P}<0.05$ compared with control; ${ }^{*} \mathrm{P}<0.05$ compared with AR.

SIRT1 expression was inhibited by adding Ly294002 (PI3K/ Akt inhibitor) to the culture or via TIM1 gene silencing, and was enhanced in $\mathrm{CD} 4^{+} \mathrm{T}$ cells from the AR group by culturing with TIM4-Ig (Fig. 3C). These results indicate that the TIM4/TIM1 interaction modulates SIRT1 expression in splenic $\mathrm{CD}^{+} \mathrm{T}$ cells during allergic inflammation via PI3K/ Akt signaling.

SIRT1 facilitates $C D 4^{+} T$-cell proliferation. To elucidate the role of SIRT1 in CD4 ${ }^{+}$T-cell proliferation, splenic DCs ( $10^{5}$ cells/well $)$ and $\mathrm{CD}^{+} \mathrm{T}$ cells $\left(10^{6}\right.$ cells/well $)$ from the AR or the control group were co-cultured in the presence of OVA (10 $\mathrm{ng} / \mathrm{ml})$ for 3 days. CD4 ${ }^{+} \mathrm{T}$ cells were labeled with CFSE or Annexin $\mathrm{V}$ for proliferation and apoptosis assessment by flow cytometry. The results demonstrated that $\mathrm{CD} 4^{+} \mathrm{T}$-cell proliferation was $\sim 9.2 \%$ in the control group, whereas it was $>40 \%$ in the AR group, which was markedly reduced by treatment with siRNA-TIM1 $(12.3 \%)$ or the addition of sirtinol (13\%), and enhanced by resveratrol stimulation (46.3\%). The proportion of $\mathrm{CD}^{+} \mathrm{T}$-cell apoptosis in the AR group (14.4\%) was higher compared with that in the control group (1.85\%), but the percentage of apoptosis by treatment with siRNA-TIM1 $(37.6 \%)$ or sirtinol $(33.3 \%)$ increased markedly, and was inhibited by treatment with resveratrol (7.45\%) (Fig. 4). These findings indicate that SIRT1 regulates $\mathrm{CD}^{+}{ }^{+} \mathrm{T}$-cell proliferation and apoptosis in allergic inflammation.
SIRT1 regulates FasL, caspase- 3 and 553 expression in $C D 4^{+}$ $T$ cells. To understand the underlying mechanisms, the expression of FasL, caspase- 3 and p53 was analyzed in CD4 ${ }^{+} \mathrm{T}$ cells. Splenic cells from the AR and control groups were cultured with OVA $(10 \mathrm{ng} / \mathrm{ml})$ for 3 days. Compared with the AR group, the addition of sirtinol $(100 \mu \mathrm{M})$ to the culture significantly increased FasL (Fig. 5), caspase-3 and p53 expression in $\mathrm{CD}^{+} \mathrm{T}$ cells, whereas treatment with resveratrol $(100 \mu \mathrm{M})$ decreased the expression of FasL, caspase-3 and p53 in CD4 ${ }^{+}$ $\mathrm{T}$ cells to the levels of the control group. These results suggest that SIRT1 downregulates the expression of FasL, caspase-3 and p53 in $\mathrm{CD}^{+} \mathrm{T}$ cells.

\section{Discussion}

Skewed Th2-cell proliferation plays a critical role in the induction of allergic inflammation, while the factors that initiate and maintain Th2-cell polarization in allergic diseases remain unclear. The TIM4/TIM1 interaction, as one of the co-stimulatory signals, is involved in the pathogenesis of allergic conditions (7-9). Using an AR mouse model, the present study demonstrated that TIM4 expression in splenic DCs was increased in AR mice, and the TIM4/TIM1 interaction promoted the expression of SIRT1 in $\mathrm{CD}^{+} \mathrm{T}$ cells during allergic inflammation via promoting PI3K/Akt phosphorylation. SIRT1 facilitated $\mathrm{CD}^{+}{ }^{+}$T-cell proliferation through downregulating 
A

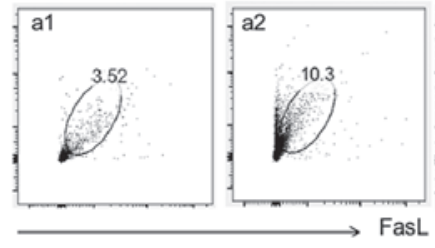

C

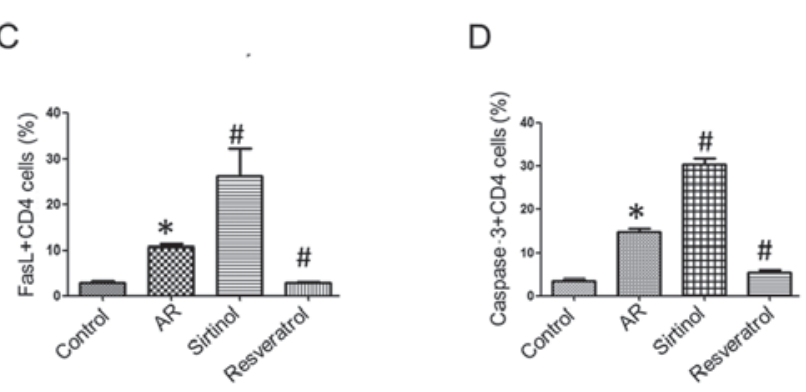

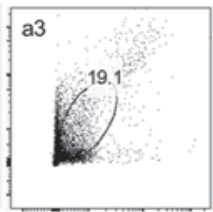
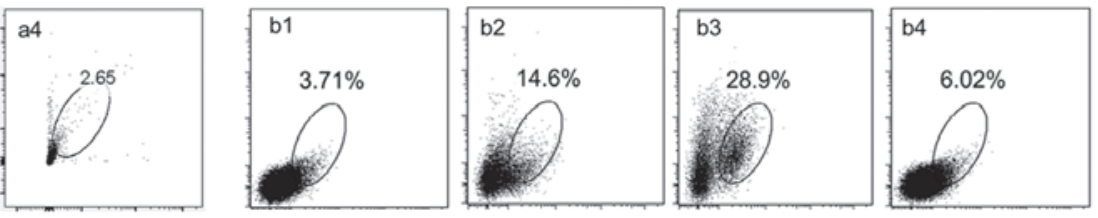

$\rightarrow$ Caspase 3
$\mathrm{E}$
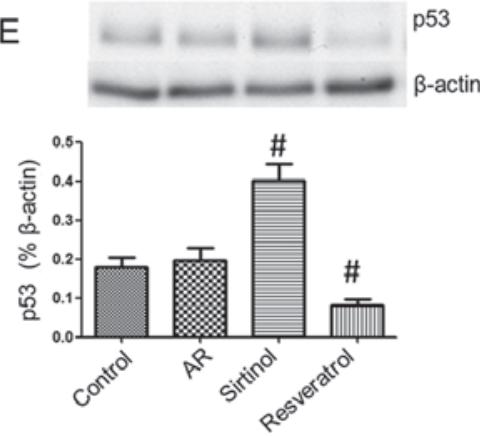

Figure 5. Silent information regulator 1 (SIRT1) regulates Fas ligand (FasL), caspase-3 and p53 expression in CD4 ${ }^{+}$T cells. (A) The gated dot plots indicate the frequency of FasL-expressing CD4 ${ }^{+} \mathrm{T}$ cells. (B) The gated dot plots indicate the frequency of caspase-3-expressing CD4 ${ }^{+} \mathrm{T}$ cells. (a1 and b1) Cells from the control group; (a2 and b2) cells from the allergic rhinitis (AR) group; (a3 and b3) cells from the AR group and CD4 ${ }^{+}$T cells were cultured with sirtinol (SIRT1 antagonist); (a4 and b4) cells from the AR group and $\mathrm{CD}^{+}{ }^{+} \mathrm{T}$ cells were stimulated with resveratrol (SIRT1 agonist). (C) The bars indicate the summarized frequencies of (A). (D) The bars indicate the summarized frequencies of (B). (E) The immune blots indicate the p53 protein levels in CD4 ${ }^{+} \mathrm{T}$ cells. The bars below the blots indicate the integrated density of the immune blots. The data are presented as mean \pm standard deviation. ${ }^{*} \mathrm{P}<0.05$ compared with control; ${ }^{\#} \mathrm{P}<0.05$ compared with $\mathrm{AR}$.

FasL, caspase-3 and p53 expression in AR mice. TIM1 is associated with the Th2 pattern of inflammation and Th2 cytokine expression; activated $\mathrm{T}$ cells expressed higher levels of TIM1 (17). TIM4, as a type I cell surface glycoprotein, is specifically expressed by APCs, such as DCs. Blockade of TIM4 on DCs repressed Th2-cell differentiation and impeded IL-4 signal transducer and activator of transcription 6 signaling (18). Consistent with these results, we observed that blockade of TIM1 or TIM4 inhibited CD4 ${ }^{+}$T-cell proliferation in AR mice. This result is supported by other studies reporting that antagonists of TIM1 blocked allergic inflammation in mouse models of asthma $(19,20)$ and allergic gut inflammation (9).

TIM1 is preferentially expressed on Th2 cells and may be upregulated following TCR stimulation (21). Stimulation of TIM4 is required by TIM1-induced T-cell proliferation (22). Previous data, as well as the present findings, indicated that TIM4/TIM1 interaction is involved in the pathogenesis of allergic diseases, while the mechanisms underlying the TIM4/TIM1 interaction in the maintenance of allergic conditions, and the precise intracellular downstream signaling by TIM1 and TIM4 engagement, remain obscure (8). TIM1 is co-localized with CD3 on the surface of T cells, and may function as part of the TCR signaling during T-cell activation, possibly through IL-2-induced ITK and PI3K phosphorylation $(8,11,23)$. SIRT1 plays an important role in cell differentiation, apoptosis and tumor suppression via post-translational modification of histone proteins, and is involved in allergic inflammation $(14,15)$. Published data demonstrated that the PI3K/Akt signaling pathway is required for the regulation of SIRT1 expression $(24,25)$. We also observed that SIRT1 mRNA expression was increased in the nasal mucosa of AR mice. The results indicate that the TIM4/TIM1 interaction modulates SIRT1 expression in splenic $\mathrm{CD}^{+} \mathrm{T}$ cells during allergic inflammation via PI3K/Akt phosphorylation.
SIRT1 is one of the deacetylases associated with asthma, and Kim et al reported that SIRT1 expression was increased in an OVA-induced murine allergic airway model, which was correlated with increased levels of IL-4, IL-5 and IL-13 and inflammatory cell infiltration in lung tissues (26). SIRT1 promotes adaptive Th2-cell responses by repressing peroxisome proliferation-activated receptor- $\gamma$ activity in DCs in an induced allergic airway mouse model (15). Our data demonstrated the role of SIRT1 in $\mathrm{CD}^{+}{ }^{+} \mathrm{T}$-cell proliferation. The findings indicated that SIRT1 enhances CD4 ${ }^{+} \mathrm{T}$-cell proliferation and inhibits their apoptosis in allergic inflammation. As a multifunctional molecule, SIRT1 is involved in a variety of molecular pathways, such as cell differentiation, cell aging and anti-inflammation. Our results revealed a novel functional aspect of SIRT1 that promotes adaptive Th2-cell responses. Other studies reported that SIRT1 plays a role in maintaining T-cell balance and exerts anti-inflammatory effects by inhibiting proinflammatory transcription factors (12). Lung SIRT1 expression decreased, while serum SIRT1 increased, in the setting of asthma (13). SIRT1 expression was reduced in the peripheral blood mononuclear cells of patients with severe asthma, and the inhibition of SIRT1 promotes a Th2-like phenotype in T cells and IL-4 gene expression via acetylation of GATA-3, but there was no correlation between IL-5 transcripts and SIRT1 activity. These inconsistent results may be due to the fact that the decrease in SIRT1 appears to be associated with oxidative stress in patients with severe asthma (14).

SIRT1 localizes in the nucleus as well as the cytoplasm and, thus, may interact with both nuclear and cytosolic proteins, and deacetylates histones and various transcription factors, such as p53 and FOXO (27). It was previously reported that SIRT1 may be a potential oncogene, which prevents apoptosis and senescence by interacting with and targeting p53 for deacetylation 
and decreasing p53-dependent transcriptional activity (12). It also suppresses FasL expression in activated T cells to interfere with activation-induced cell death (AICD) (28). Caspase-3 plays an important role in the induction of cell apoptosis (29). Our previous study suggested that Fas/FasL, p53 and caspase-3 are involved in the course of CD4 ${ }^{+} \mathrm{T}$-cell apoptosis and AICD (30). In the present study, to elucidate the mechanisms underlying SIRT1 regulation of $\mathrm{CD}^{+}{ }^{+} \mathrm{T}$-cell proliferation in allergic inflammation, the expression of FasL, caspase-3 and p53 was determined in $\mathrm{CD}^{+} \mathrm{T}$ cells. The results suggested that SIRT1 downregulates FasL, caspase- 3 and p53 expression in CD4 ${ }^{+} \mathrm{T}$ cells.

In conclusion, the present study demonstrated that the TIM4/TIM1 interaction promotes PI3K/Akt phosphorylation in $\mathrm{CD}^{+}{ }^{+} \mathrm{T}$ cells, resulting in increased SIRT1 expression; SIRT1 then facilitates $\mathrm{CD}^{+} \mathrm{T}$-cell proliferation through downregulating FasL, caspase-3 and p53 expression in AR mice. These results suggest that the TIM4/TIM1 interaction modulates Th2-cell inflammation through enhancing SIRT1 expression.

\section{Acknowledgements}

The present study was supported by grants from the Natural Science Foundation of China (no. 81571790), the Innovation of Science and Technology Commission of Shenzhen Municipality (nos. JCYJ20140411150916749, JCYJ20160429091935720, ZDSYS201506050935272 and YLWS20140609111127924), the Medical Science and Technology Research Fund of Guangdong province (A2016272; no. 2014A030313781), and the Health Committee Foundation of Shenzhen (nos. 201401097 and 201401096). The study was also supported by a grant from the Innovation of Science and Technology Commission of Shenzhen Municipality (no. JCYJ20150403091931195).

\section{References}

1. Sicherer SH and Sampson HA: Food allergy: Epidemiology, pathogenesis, diagnosis, and treatment. J Allergy Clin Immunol 133: 291-307, quiz 308, 2014.

2. Heratizadeh A: Atopic dermatitis: New evidence on the role of allergic inflammation. Curr Opin Allergy Clin Immunol 16: 458-464, 2016.

3. Rosati MG and Peters AT: Relationships among allergic rhinitis, asthma, and chronic rhinosinusitis. Am J Rhinol Allergy 30: 44-47, 2016.

4. Eifan $\mathrm{AO}$ and Durham SR: Pathogenesis of rhinitis. Clin Exp Allergy 46: 1139-1151, 2016.

5. Oettgen HC and Burton OT: IgE receptor signaling in food allergy pathogenesis. Curr Opin Immunol 36: 109-114, 2015.

6. Hiraishi Y, Nambu A, Shibui A, Nakanishi W, Yamaguchi S, Morita H, likura M, McKenzie AN, Matsumoto K, Sudo K, et al: TIM-3 is not essential for development of airway inflammation induced by house dust mite antigens. Allergol Int 65: 459-465, 2016

7. Li Z, Ju Z and Frieri M: The T-cell immunoglobulin and mucin domain (Tim) gene family in asthma, allergy, and autoimmunity. Allergy Asthma Proc 34: e21-e26, 2013.

8. Freeman GJ, Casasnovas JM, Umetsu DT and DeKruyff RH: TIM genes: A family of cell surface phosphatidylserine receptors that regulate innate and adaptive immunity. Immunol Rev 235: 172-189, 2010.

9. Feng BS, Chen X, He SH, Zheng PY, Foster J, Xing Z, Bienenstock $J$ and Yang PC: Disruption of T-cell immunoglobulin and mucin domain molecule (TIM)-1/TIM4 interaction as a therapeutic strategy in a dendritic cell-induced peanut allergy model. J Allergy Clin Immunol 122: 55-61.e1-7, 2008.

10. Santiago C, Ballesteros A, Tami C, Martínez-Muñoz L, Kaplan GG and Casasnovas JM: Structures of T Cell immunoglobulin mucin receptors 1 and 2 reveal mechanisms for regulation of immune responses by the TIM receptor family. Immunity 26: 299-310, 2007.
11. Binne LL, Scott ML and Rennert PD: Human TIM-1 associates with the TCR complex and up-regulates T cell activation signals. J Immunol 178: 4342-4350, 2007.

12. Haigis MC and Sinclair DA: Mammalian sirtuins: Biological insights and disease relevance. Annu Rev Pathol 5: 253-295, 2010.

13. Wang Y, Li D, Ma G, Li W, Wu J, Lai T, Huang D, Zhao X, Lv Q, Chen M, et al: Increases in peripheral SIRT1: A new biological characteristic of asthma. Respirology 20: 1066-1072, 2015.

14. Colley T, Mercado N, Kunori Y, Brightling C, Bhavsar PK, Barnes PJ and Ito K: Defective sirtuin-1 increases IL-4 expression through acetylation of GATA-3 in patients with severe asthma. J Allergy Clin Immunol 137: 1595-1597.e7, 2016.

15. Legutko A1, Marichal T, Fiévez L, Bedoret D, Mayer A, de Vries H, Klotz L, Drion PV, Heirman C, Cataldo D, et al: Sirtuin 1 promotes Th2 responses and airway allergy by repressing peroxisome proliferator-activated receptor-gamma activity in dendritic cells. J Immunol 187: 4517-4529, 2011.

16. Kim YH, Yang TY, Park CS, Ahn SH, Son BK, Kim JH, Lim DH and Jang TY: Anti-IL-33 antibody has a therapeutic effect in a murine model of allergic rhinitis. Allergy 67: 183-190, 2012.

17. de Souza AJ, Oriss TB, O'malley KJ, Ray A and Kane LP: T cell Ig and mucin 1 (TIM-1) is expressed on in vivo-activated T cells and provides a costimulatory signal for $\mathrm{T}$ cell activation. Proc Natl Acad Sci USA 102: 17113-17118, 2005.

18. Li J, Zhao X, Liu X and Liu H: Disruption of TIM-4 in dendritic cell ameliorates hepatic warm IR injury through the induction of regulatory T cells. Mol Immunol 66: 117-125, 2015.

19. Sonar SS, Hsu YM, Conrad ML, Majeau GR, Kilic A, Garber E, Gao Y, Nwankwo C, Willer G, Dudda JC, et al: Antagonism of TIM-1 blocks the development of disease in a humanized mouse model of allergic asthma. J Clin Invest 120: 2767-2781, 2010.

20. Kim HY, Chang YJ, Chuang YT, Lee HH, Kasahara DI, Martin T, Hsu JT, Savage PB, Shore SA, Freeman GJ, et al: T-cell immunoglobulin and mucin domain 1 deficiency eliminates airway hyperreactivity triggered by the recognition of airway cell death. J Allergy Clin Immunol 132: 414-425.e6, 2013.

21. Yeung MY, McGrath M and Najafian N: The emerging role of the TIM molecules in transplantation. Am J Transplant 11: 2012-2019, 2011.

22. Mariat C, Degauque N, Balasubramanian S, Kenny J, DeKruyff RH, Umetsu DT, Kuchroo V, Zheng XX and Strom TB. Tim-1 signaling substitutes for conventional signal 1 and requires costimulation to induce $\mathrm{T}$ cell proliferation. J Immunol 182: 1379-1385, 2009.

23. de Souza AJ, Oak JS, Jordanhazy R, DeKruyff RH, Fruman DA and Kane LP: T cell Ig and mucin domain-1-mediated T cell activation requires recruitment and activation of phosphoinositide 3-kinase. J Immunol 180: 6518-6526, 2008

24. Koga T, Suico MA, Shimasaki S, Watanabe E, Kai Y, Koyama K, Omachi K, Morino-Koga S, Sato T, Shuto T, et al: Endoplasmic reticulum (ER) stress induces sirtuin 1 (SIRT1) expression via the PI3K-Akt-GSK3 $\beta$ signaling pathway and promotes hepatocellular injury. J Biol Chem 290: 30366-30374, 2015.

25. Ming GF, Tang YJ, Hu K, Chen Y, Huang WH and Xiao J: Visfatin attenuates the ox-LDL-induced senescence of endothelial progenitor cells by upregulating SIRT1 expression through the PI3K/Akt/ERK pathway. Int J Mol Med 38: 643-649, 2016.

26. Kim SR, Lee KS, Park SJ, Min KH, Choe YH, Moon H, Yoo WH, Chae HJ, Han MK and Lee YC: Involvement of sirtuin 1 in airway inflammation and hyperresponsiveness of allergic airway disease. J Allergy Clin Immunol 125: 449-460.e14, 2010.

27. Tanno M, Sakamoto J, Miura T, Shimamoto K and Horio Y: Nucleocytoplasmic shuttling of the $\mathrm{NAD}^{+}$-dependent histone deacetylase SIRT1. J Biol Chem 282: 6823-6832, 2007.

28. Arakaki R, Yamada A, Kudo Y, Hayashi Y and Ishimaru N: Mechanism of activation-induced cell death of T cells and regulation of FasL expression. Crit Rev Immunol 34: 301-314, 2014.

29. Porter AG and Jänicke RU: Emerging roles of caspase-3 in apoptosis. Cell Death Differ 6: 99-104, 1999.

30. Yang L, Xu LZ, Liu ZQ, Yang G, Geng XR, Mo LH, Liu ZG, Zheng PY and Yang PC: Interleukin-13 interferes with activationinduced T-cell apoptosis by repressing p53 expression. Cell Mol Immunol 13: 669-677, 2016. 